\title{
Inflammatory potential of diet and risk of incident knee osteoarthritis: a prospective cohort study
}

\author{
Qiang Liu',2, James R. Hebert ${ }^{3,4}$, Nitin Shivappa ${ }^{3,4}$, Jianjun Guo ${ }^{5}$, Ke Tao ${ }^{1}$, Chao Zeng ${ }^{6}$, Guanghua Lei ${ }^{6}$, \\ Jianhao Lin $^{1 *}$ and Yuqing Zhang ${ }^{2^{*}}$
}

\begin{abstract}
Background: To examine the relation between inflammatory potential of diet and incident knee osteoarthritis (OA) and the role of BMI in the association of interest.

Methods: In the Osteoarthritis Initiative, the energy-adjusted dietary inflammatory index (E-DIIM) scores were calculated based on the Block Brief 2000 Food Frequency Questionnaire and categorized into sex-specific quartiles. Outcomes were incident (1) radiographic knee OA (ROA) (i.e., a KL grade $\geq 2$ ) and (2) symptomatic knee OA (SxOA) (i.e., a combination of frequent knee pain and ROA). We fitted generalized estimating equation models to examine the association between E-DII scores and incident knee OA. We performed mediation analyses to assess the potential mediation by BMI in the DII-OA relation.

Results: Over a 48-month follow-up period, 232 and 978 knees developed ROA and SxOA, respectively. Compared with the lowest (most anti-inflammatory) E-DIl quartile, the odds ratio (OR) of incident ROA for the highest (most pro-inflammatory) E-DII quartile was 1.73 (95\% confidence interval $(\mathrm{Cl}) 1.15$ to $2.62, P_{\text {trend }}=0.007$ ). The corresponding OR for SxOA was $1.43\left(95 \% \mathrm{Cl} 1.16\right.$ to $\left.1.76, P_{\text {trend }}=0.001\right)$. The DII-OA association was significantly mediated via BMI with an indirect effect of $1.08(95 \% \mathrm{Cl} 1.04,1.13)$ for $\mathrm{ROA}$ and $1.13(95 \% \mathrm{Cl} 1.09,1.16)$ for SxOA, accounting for $20.4 \%$ and $44.5 \%$ of the total effect, respectively.
\end{abstract}

Conclusions: A higher inflammatory potential of diet increased the risk of knee OA. The association was significantly mediated via BMI. Targeting the inflammatory potential of diet may be beneficial to reduce the risk of knee OA.

Keywords: Osteoarthritis, Diet, Inflammation, Body mass index, Cohort

\section{Background}

Diet plays a fundamental role in preventing many chronic diseases $[1,2]$. One of the mechanisms is through regulating inflammation [3-5]. Indeed, the inflammatory potential of diet has been shown to be associated with obesity $[6,7]$ and cardiovascular diseases $[8,9]$, two conditions

\footnotetext{
*Correspondence: linjianhao@pkuph.edu.cn; yzhang108@mgh.harvard.edu ${ }^{1}$ Arthritis Clinic and Research Center, Peking University People's Hospital, No.11 Xizhimen South Road, Xicheng District, Beijing 100044, China

${ }^{2}$ Division of Rheumatology, Allergy and Immunology, Massachusetts General Hospital, Harvard Medical School, 55 Fruit St., Boston, MA 02114, USA Full list of author information is available at the end of the article
}

well known to be related to chronic, systemic inflammation. Osteoarthritis (OA), a common form of arthritis that is pathologically associated with biomechanics and inflammation [10], is highly prevalent among the elderly and is a leading cause of disability [11, 12]. A limited number of risk factors for OA are modifiable [13]. Diet can be modified on a daily basis, and the evidence for its role in OA development is accumulating $[14,15]$. Despite the role of the whole diet in determining health outcomes, a majority of studies have focused on single dietary components. Complex human diets consist of multiple components

(c) The Author(s). 2020 Open Access This article is licensed under a Creative Commons Attribution 4.0 International License, which permits use, sharing, adaptation, distribution and reproduction in any medium or format, as long as you give appropriate credit to the original author(s) and the source, provide a link to the Creative Commons licence, and indicate if changes were made. The images or other third party material in this article are included in the article's Creative Commons licence, unless indicated otherwise in a credit line to the material. If material is not included in the article's Creative Commons licence and your intended use is not permitted by statutory regulation or exceeds the permitted use, you will need to obtain permission directly from the copyright holder. To view a copy of this licence, visit http://creativecommons.org/licenses/by/4.0/. The Creative Commons Public Domain Dedication waiver (http://creativecommons.org/publicdomain/zero/1.0/) applies to the data made available in this article, unless otherwise stated in a credit line to the data. 
that interact with one another and exert inflammatory potential as a whole; thus, it is reasonable to use a summary measure to quantify the inflammatory potential of diet as a whole. In this sense, a dietary index that summarizes the functional effect of food has an advantage over examining single dietary components in the study of diet-disease associations [16]. To the best of our knowledge, no studies have used a summary measure that quantifies the inflammatory potential of diet to examine the association of diet with the risk of incident OA.

High body mass index (BMI) is a well-established risk factor for OA and is associated with both biomechanics and inflammation in OA pathology [10, 17]. Diet plays a central role in determining BMI. At its simplest, diet provides the energy intake side of the energy balance equation. Reflecting the nuanced way that diet can exert an effect on body mass, a higher inflammatory potential of diet as measured by the dietary inflammatory index (DII) has been shown, prospectively, to increase the risk of weight gain and incident obesity [6]. Despite the interplay between diet, $\mathrm{BMI}$, and $\mathrm{OA}$, the role of $\mathrm{BMI}$ in the diet-OA association remains largely unexplored. There is evidence that BMI can mediate the association of diet with serum inflammatory markers on the basis of its inflammatory potential [18]. For example, a recent study showed that adjustment for BMI substantially attenuated the association of dietary fiber with the risk of symptomatic knee OA (SxOA) [19]. Taken together, these findings suggest that BMI may function as a mediator in the association between the inflammatory potential of diet and knee OA. However, such a hypothesis has not been formally tested yet.

To fill in these gaps of knowledge, we conducted a longitudinal cohort study using data from the Osteoarthritis Initiative (OAI). We aimed to examine (1) the association of inflammatory potential of diet with the risk of incident knee OA and (2) the mediation effect of BMI in the association of interest.

\section{Methods}

\section{Study population}

The OAI is a multi-center, longitudinal prospective cohort study. At baseline, 4796 US men and women (58.5\%) age 47-79 years with, or at high risk of, knee $\mathrm{OA}$ were recruited from four sites: Columbus, $\mathrm{OH}$; Providence, RI; Baltimore, MD; and Pittsburgh, PA. Annual radiographic assessments of knee OA were carried out until the 48-month OAI follow-up visit; therefore, in the present study, we followed the participants until 48 months.

Baseline assessment of inflammatory potential of diet Habitual dietary intake of nutrients and foods was estimated at baseline using a validated dietary assessment tool, the Block Brief 2000 Food Frequency Questionnaire (FFQ) [20]. For each dietary component, the frequency of consumption was reported according to nine predetermined categories ranging from "never" to "everyday" with illustrations of standard portion sizes. Dietary intake of each component and energy was calculated based on nutrient composition values determined from the US Department of Agriculture nutrient database [21].

The DII, developed by Cavicchia et al. [22] and updated by Shivappa et al. [23], is a literature-derived population-based scoring algorithm to assess the inflammatory potential of diet as a whole based on 45 food parameters. A higher DII score indicates a greater inflammatory potential of diet (i.e., pro-inflammatory effect). Details regarding the development and calculation process are available elsewhere [23]. The DII has been evaluated for validity in 27 studies and shown to predict inflammatory markers including $\mathrm{C}$-reactive protein (CRP), interleukin (IL)-6, and tumor necrosis factor (TNF) $\alpha[22,24-27]$. Using data collected by the FFQ in the OAI, we calculated the energy-adjusted DII (E-DII ${ }^{\mathrm{mw}}$ ) based on the intake of 24 dietary components, defined as DII score per $4184 \mathrm{~kJ}$ (1000 kcal) of energy [28]. Dietary components available in the OAI for calculating E-DII scores included vitamin B12, vitamin B6, $\beta$-carotene, caffeine, carbohydrate, cholesterol, fat, fiber, folic acid, iron, magnesium, monounsaturated fat acids, niacin, protein, polyunsaturated fatty acids, riboflavin, saturated fat acids, selenium, thiamin, vitamin $\mathrm{A}$, vitamin $\mathrm{C}$, vitamin $\mathrm{E}$, vitamin $\mathrm{D}$, and zinc.

\section{Assessment of incident knee OA outcomes}

At baseline and at each annual follow-up visit, frequent knee pain was queried and defined as pain, aching, or stiffness for more than half the days of a month during the past 12 months. Participants obtained weight-bearing fixed-flexion posterior-anterior view radiographs of both knees. Central reading was carried out to assess Kellgren and Lawrence (KL) grade for each knee. Any disagreement as to whether the knee at any time point had radiographic OA was adjudicated by a panel of three experienced readers including the two primary readers and one other. We defined a knee as having incident radiographic knee OA (ROA) if it did not have ROA at baseline (i.e., $\mathrm{K} / \mathrm{L}=0$ or 1 ) and developed $\mathrm{ROA}$ (i.e., $\mathrm{K} / \mathrm{L} \geq 2$ ) over the follow-up time. Incident knee SxOA was defined as a new onset of a combination of frequent knee pain and ROA in the same knee during the follow-up period.

\section{Assessment of other covariates and BMI as a potential mediator}

At baseline, all participants were queried for age, sex, race, educational attainment, annual income, and 
tobacco use. Physical activity was assessed using the Physical Activity Scale for the Elderly (PASE) capturing a broad spectrum of habitual physical activity and is summarized into a continuous score with higher scores indicating higher levels of physical activity. PASE was validated for the assessment of physical activity among older adults with knee pain and physical disability [29]. At the 12-month follow-up visit, body weight and height were measured in light clothing without shoes using calibrated devices. BMI was calculated using weight $(\mathrm{kg}) \mathrm{di}-$ vided by the square of height $(\mathrm{m})$. We used BMI assessed at the 12-month follow-up visit as the mediator in the current study.

\section{Statistical analysis}

We categorized E-DII scores into quartiles for men and women separately to account for sex differences in dietary intake of nutrients and foods. We examined the association of E-DII with the risk of knee ROA (or SxOA) by comparing higher E-DII quartiles (Q2, Q3, and Q4) with the lowest quartile (Q1, reference category) using generalized estimating equations (GEE) to account for the correlation between two knees for each participant. In the base model (model 1), we adjusted for age, sex (men vs women), race (White vs non-White), and total energy intake ( $\mathrm{kcal} /$ day). In model 2 , we further adjusted for educational attainment (below college vs college or above), annual income (<50,000 US\$ vs $\geq 50,000$ US\$), tobacco use (non-smoker vs former and current smoker), and PASE score. We tested for linear trend using the median value of each quartile of E-DII score as a continuous variable in the regression model. We also conducted alternative analyses fitting E-DII as a continuous variable in model 1 and model 2 . We conducted sensitivity analyses by counting total knee replacement due to knee OA during the follow-up period as incident knee ROA or SxOA.
We performed mediation analyses to assess to what extent the association of E-DII score with incident ROA (or SxOA) was mediated through BMI assessed at the 12-month follow-up visit. In these analyses, we grouped the E-DII score into two categories using sex-specific median value as a cut-point. We decomposed the total effect of E-DII score on the risk of incident knee ROA (or SxOA) into two components [30, 31] (Fig. 1), i.e., (1) the indirect effect (or mediated effect) representing the effect of E-DII on the risk of incident knee ROA (or SxOA) mediated via BMI and (2) the direct effect representing the effect of E-DII score on the risk of incident knee ROA (or SxOA) that was not through BMI. We calculated the proportion of mediation to quantify the proportion of the effect of E-DII mediated by BMI [30, 31].

All statistical analyses were performed using Stata/SE 15.1 (StataCorp, TX, USA). A $P$ value $<0.05$ (two-sided) was considered statistically significant.

\section{Results}

We included 1786 and 2940 eligible participants in the final analyses for the association of E-DII score with incident knee ROA and SxOA, respectively. The inclusion of subjects and knees is described in Fig. 2.

We summarized the baseline characteristics according to sex-specific quartiles of E-DII score in Table 1 . Among participants included in the analysis of incident ROA or SxOA, subjects in the highest E-DII quartile (Q4, reflecting the most pro-inflammatory diet) were younger, more likely to have attained college or higher educational level, and had an annual income $\geq 50,000$ US\$ as well as a higher total energy intake and BMI than their counterparts in the lowest quartile of E-DII (Q1, representing the most anti-inflammatory diet).

As shown in Table 2, a higher E-DII score was associated with an increased risk of incident ROA in both the

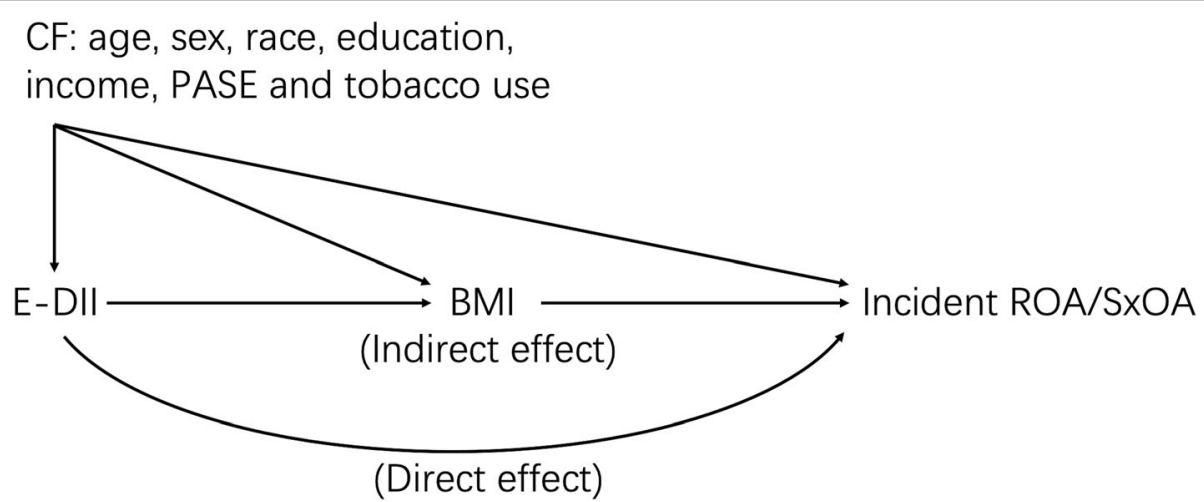

Fig. 1 A directed acyclic graph for the decomposition of the total effect into direct and indirect effect. E-DII, energy-adjusted dietary inflammatory index; ROA, radiographic knee osteoarthritis; SxOA, symptomatic knee osteoarthritis; CF, confounder; PASE, Physical Activity Scale for the Elderly; BMI, body mass index 


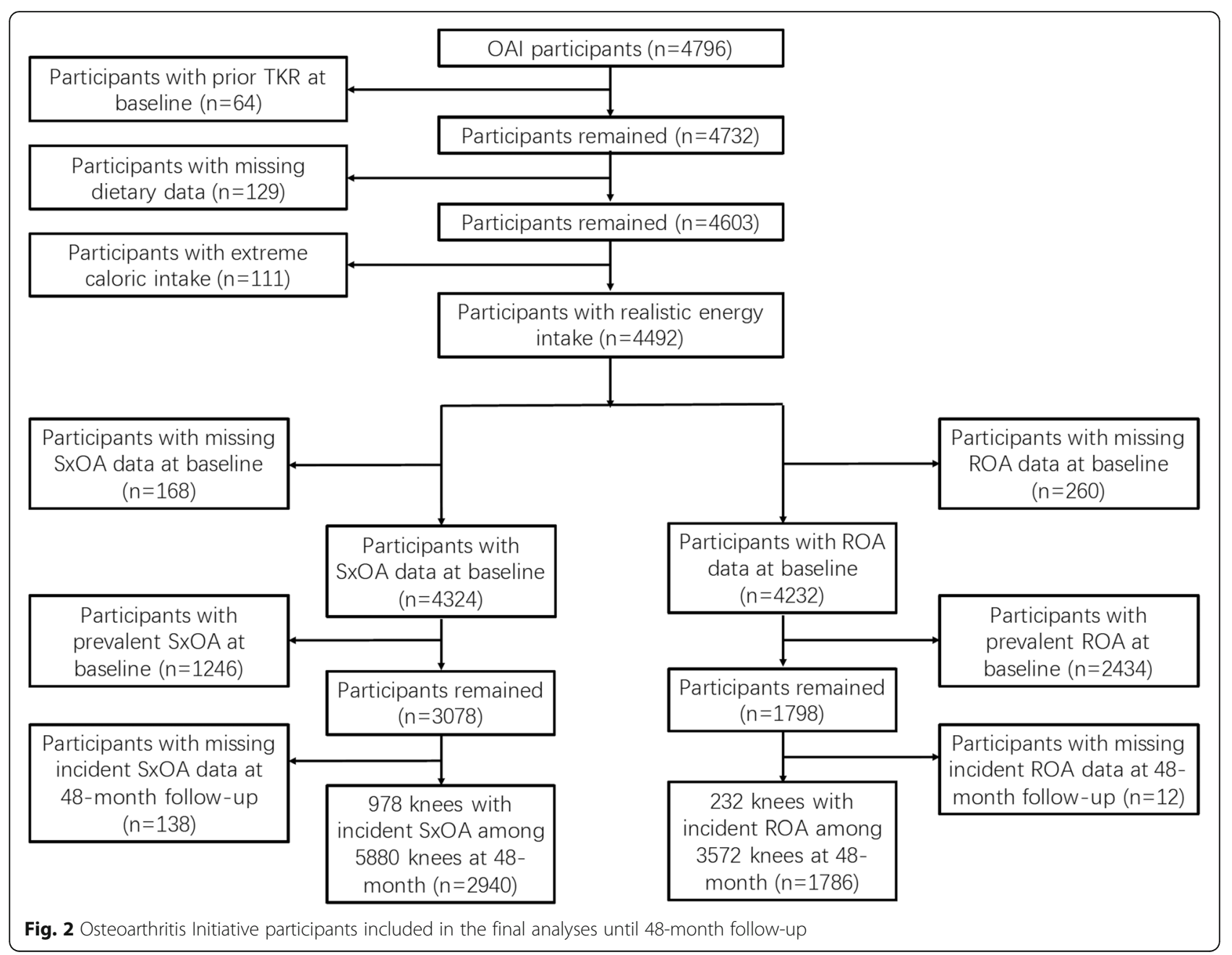

base model and fully adjusted model. Compared with subjects in Q1, those in Q4 had a higher risk of incident ROA with an odds ratio (OR) of 1.73 (95\% confidence interval (CI) 1.15 to 2.62). Test for the linear trend of EDII score with the risk of incident ROA was statistically significant $(P$ for trend $=0.007)$. A similar association between E-DII score and incident SxOA also was observed. Subjects in Q4 had a significantly higher risk of incident SxOA than their counterparts in Q1 $(\mathrm{OR}=1.43,95 \% \mathrm{CI}$ 1.16 to $1.76, P$ for trend $=0.001$ ). When we fitted E-DII as a continuous variable in both the base model and fully adjusted model, the association of E-DII with ROA (OR from the fully adjusted model $=1.09,95 \%$ CI $1.01,1.17$ ) and SxOA (OR from the fully adjusted model $=1.06$, $95 \%$ CI 1.02, 1.11) remained statistically significant. When total knee replacement due to knee OA was counted as an incident case of either ROA or SxOA, the results did not change materially.

The total, natural direct, and indirect effects of E-DII score on the risk of incident ROA mediated via BMI are shown in Table 3 . BMI significantly mediated the effect of E-DII on incident ROA, comparing participants with an E-DII < median (i.e., 2.50) with those whose E-DII $\geq$ median (OR for indirect effect $=1.08,95 \%$ CI 1.04 to $1.13)$, with a proportion of mediation of $20.4 \%$. The direct effect of E-DII on incident ROA not through BMI was statistically significant ( $\mathrm{OR}=1.36,95 \% \mathrm{CI} 1.02$ to 1.82). Similarly, the indirect effect (i.e., mediated effect) of E-DII on incident SxOA through BMI was 1.13 (95\% CI 1.09 to 1.16 ) while the direct effect $(\mathrm{OR}=1.16,95 \%$ CI 1.01, 1.34) also was statistically significant. About $44.5 \%$ of the association between E-DII score and the risk of incident SxOA was mediated via BMI.

\section{Discussion}

In this prospective US cohort, we found that a higher EDII score was associated with an increased risk of incident ROA and SxOA after controlling for several potential confounders including total energy intake. The impact of the DII on incident knee ROA and SxOA was, in part, due to its effect on BMI. 


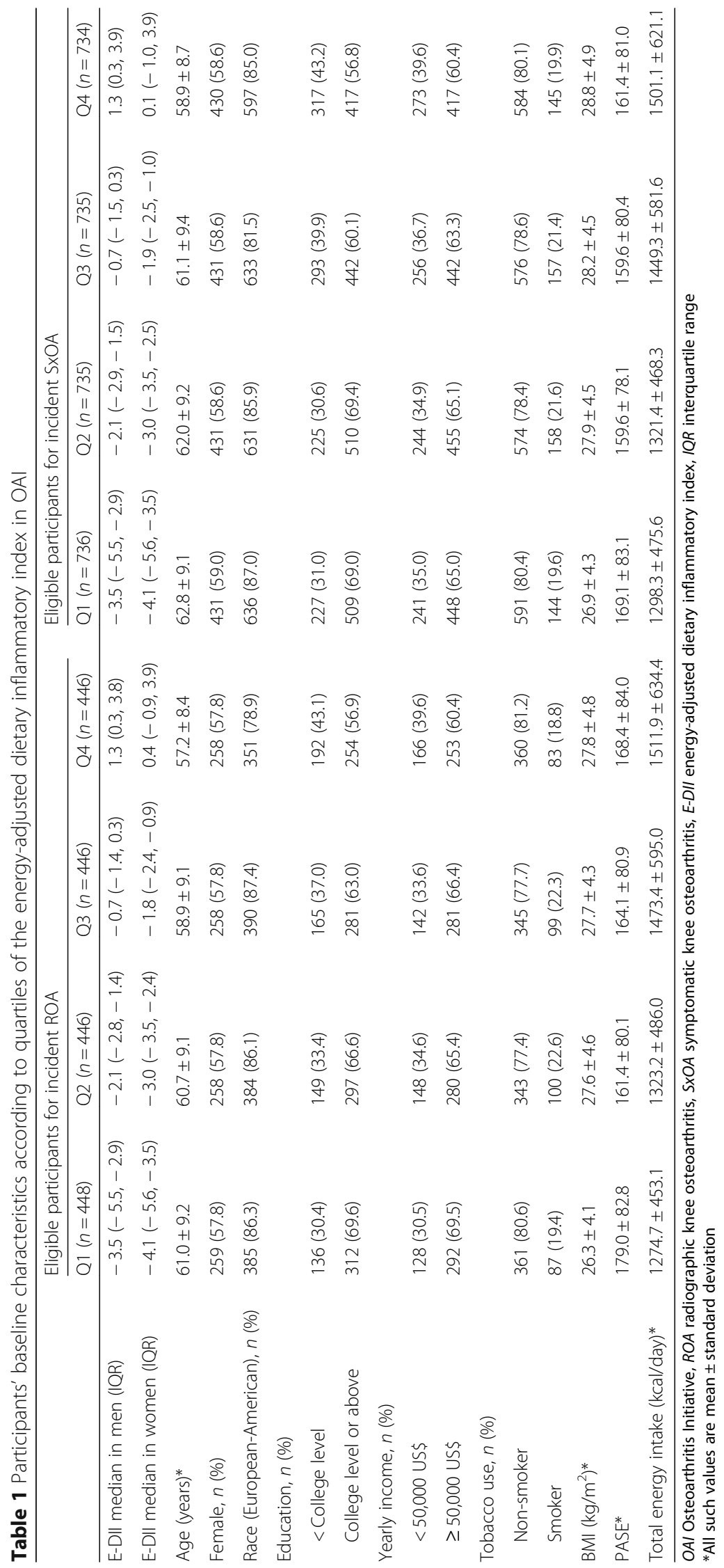


Table 2 Total effect of E-DII on incident ROA and incident SxOA

\begin{tabular}{|c|c|c|c|c|c|}
\hline E-DII in quartile & Q1 & Q2 & Q3 & Q4 & $P$ for trend ${ }^{*}$ \\
\hline \multicolumn{6}{|l|}{ ROA } \\
\hline Median & -3.9 & -2.7 & -1.3 & 0.9 & \\
\hline ROA knees" & $48 / 896$ & $53 / 892$ & $66 / 892$ & $65 / 892$ & \\
\hline Model $1^{\dagger}$ & 1.00 (ref) & $1.12(0.75,1.68)$ & $1.48(1.00,2.18)$ & $1.43(0.96,2.11)$ & 0.049 \\
\hline Model $2^{\ddagger}$ & 1.00 (ref) & $1.27(0.84,1.94)$ & $1.53(1.01,2.32)$ & $1.73(1.15,2.62)$ & 0.007 \\
\hline \multicolumn{6}{|l|}{$\mathrm{SxOA}$} \\
\hline Median & -3.9 & -2.7 & -1.4 & 0.8 & \\
\hline SxOA knees\# & $218 / 1472$ & $237 / 1470$ & $260 / 1470$ & $263 / 1468$ & \\
\hline Model $1^{\dagger}$ & 1.00 (ref) & $1.12(0.92,1.37)$ & $1.28(1.05,1.56)$ & $1.37(1.12,1.68)$ & 0.001 \\
\hline Model $2^{\ddagger}$ & 1.00 (ref) & $1.13(0.92,1.40)$ & $1.27(1.04,1.56)$ & $1.43(1.16,1.76)$ & 0.001 \\
\hline
\end{tabular}

E-DIl energy-adjusted dietary inflammatory index, $B M I$ body mass index, OA osteoarthritis, PASE Physical Activity in the Elderly Scale, ROA radiographic knee osteoarthritis, SxOA, symptomatic knee osteoarthritis

*Test for trend based on the variable containing the median value for each quartile

\#Number of OA affected/total number of knees in each quartile of the dietary inflammatory index

${ }^{\dagger}$ Model 1 adjusted for age (years), sex (men vs women), race (white vs non-white), and total energy intake (kcal/day)

${ }^{\ddagger}$ Model 2 further adjusted for education (< college vs $\geq$ college), yearly income level (<50,000 US\$ vs $\geq 50,000$ US\$), tobacco use (non-smoker vs smoker), and physical activity (PASE, continuous)

Observational studies on single dietary components have failed to be corroborated in randomized trials. For example, supplements of vitamin $\mathrm{E}$ were ineffective while vitamin D did not achieve a clinically important effect on knee pain in OA compared with placebo [32]. However, people do not consume nutrients or foods in isolation. The importance of accounting for the effect of diet as a whole has been emphasized increasingly over the past several years [1, $2,16,33]$. Using a dietary index to quantify the inflammatory potential of the whole diet may increase the robustness and validity in detecting the relation to disease [16]. The DII and, by logical extension, the E-DII account for a variety of nutrients and foods for

Table 3 Direct and indirect effects of E-DII on incident ROA and SxOA for mediation via BMI

\begin{tabular}{lll}
\hline E-DIl & $<2.50$ & $\geq 2.50$ \\
\hline ROA & $n=894$ persons & $n=892$ persons \\
Total effect* & 1.00 (ref) & $1.47(1.10,1.97)$ \\
Direct effect & 1.00 (ref) & $1.36(1.02,1.82)$ \\
Indirect effect via BMI & 1.00 (ref) & $1.08(1.04,1.13)$ \\
Proportion of mediation via BMI & $20.4 \%$ & \\
SxOA & $n=1471$ persons & $n=1469$ persons \\
Total effect* & 1.00 (ref) & $1.31(1.13,1.51)$ \\
Direct effect & 1.00 (ref) & $1.16(1.01,1.34)$ \\
Indirect effect via BMl & 1.00 (ref) & $1.13(1.09,1.16)$ \\
Proportion of mediation via BMl & $44.5 \%$ & \\
\hline
\end{tabular}

E-DIl energy-adjusted dietary inflammatory index, $B M I$ body mass index, $R O A$ radiographic knee osteoarthritis, $S x O A$ symptomatic knee osteoarthritis *Model adjusted for age (years), sex (men vs women), race (white vs nonwhite), total energy intake ( $\mathrm{kcal} /$ day), education (< college vs $\geq$ college), yearly income level ( $<50,000$ US\$ vs $\geq 50,000$ US\$), tobacco use (non-smoker vs smoker), and physical activity (PASE, continuous) which evidence indicates the ability to exert pro- or anti-inflammatory effect within a diet [23]. Our study, as an advance over previous observational studies focusing on single dietary components, is the first one to use a dietary index to examine the association between diet and incident knee OA. Our findings, if confirmed in other, larger prospective studies, may inform a prevention strategy, with more emphasis on the effect of diet as a whole, for this highly prevalent disabling disease.

There is evidence that a higher DII score increases the risk of weight gain and incident obesity, independent of total energy intake [7]. Thus, an intuitive question is: To what extent the DII-OA association is mediated via BMI with adjustment for total energy intake? Indeed, we found the effect of the E-DII on incident knee OA was substantially mediated through BMI as evidenced by a proportion of mediation of $20.4 \%$ for ROA and $44.5 \%$ for $\mathrm{SxOA}$, which is independent of total energy intake. Weight loss reduces the risk of symptomatic knee OA [34]. Dietary strategies to lose weight have primarily focused on limiting total energy intake [35-37]. Our findings, along with those of previous studies [6,7], suggest that a diet with low inflammatory potential may be synergistic in helping individuals to reduce the risk of incident knee OA. Because diet can affect inflammation through a variety of dietary parameters, its effect is not limited to restricting total energy intake. It is plausible to take inflammatory potential of diet into consideration in dietary strategies for people at high risk of developing knee OA. Nevertheless, observational studies can only provide empirical evidence; thus, randomized clinical trials may be warranted to test this hypothesis. However, it is important to understand the nuances of intervening in dietary behaviors [38]. 
Inflammation plays an integrated role in OA pathology $[39,40]$. Diet regulates inflammation [3-5, 41, 42]. Inflammatory potential of diet, as quantified by DII and the E-DII, predicts circulating inflammatory markers such as IL-6, TNF- $\alpha$, and CRP [22, 24-27]. It has been shown that these inflammatory markers are associated with knee pain and joint damage [43-45]. In our study, mediation analyses showed that the direct effect of the E-DII on incident knee ROA or SxOA was statistically significant, suggesting the association between E-DII score and the risk of knee OA may not be entirely accounted by its effect on BMI. The DII-OA relation also may be mediated via inflammatory markers. However, due to a lack of data on circulating inflammatory markers in OAI, we were not able to examine this potential causal pathway of DII-OA. Further studies are needed to address this question. These must be designed to elucidate the temporal order of effects, an important criterion for assessing causality [46, 47]. Furthermore, these studies must account for the knowledge that diet can exert a direct effect on inflammation (i.e., not mediated via BMI or adiposity) as well as its role in affecting energy balance $[6,48,49]$.

There are notable strengths of this study. We used data from OAI, a well-executed multi-center longitudinal cohort study with a large sample. The exposure, mediator, and outcomes in our study were assessed using validated instruments and standard protocols. Central reading of knee radiographs blinded to the chronological order of follow-up with consensus adjudication is especially appreciated to improve the reliability of incident knee OA assessment. We were able to account for the intraclass correlation between knees within individuals using the GEE approach. This is important because individuals tend to get bilateral $\mathrm{OA}$ of the knee [50]. The DII/E-DII is a well-validated tool that can be applied in any population with a wide range of data source [23, 24]. The use of objective measurements of body weight and height reduces potential biases. Furthermore, E-DII scores were associated with both incident ROA and SxOA. Consistent findings lend additional validity to our study.

Our findings should be interpreted with caution. First, only 24 out of 45 food parameters were available for calculating E-DII scores in the OAI, resulting in a lessthan-ideal assessment of the inflammatory potential of diet. However, the predictive ability of the DII has been shown to be relatively preserved in calculations using < 30 parameters [24]. Moreover, the FFQ covered a majority of dietary components that have been related to OA in the literature $[14,15]$, with eight out of nine proinflammatory food parameters accounted for in calculating DII/E-DII [23]. Second, given dietary intakes were queried only once at the baseline visit, misclassifications may occur due to natural, seasonal fluctuations in dietary intake and secular trends in dietary change. Nevertheless, such exposure misclassification, if occurred, is likely to be non-differential and would bias the effect estimate towards the null. Third, physical activity was assessed only using the self-reported PASE questionnaire at baseline visit in the OAI, which may not be as accurate and reliable as that by objective measures. However, the PASE questionnaire has been validated for the assessment of physical activity among adults with knee pain and disability [29]. Finally, our findings may not be fully generalizable to other populations due to the selection of participants who were at high risk of knee OA in OAI. Other factors, such as genetic background and environmental exposures, also should be taken into consideration when interpreting our findings.

\section{Conclusions}

Greater dietary inflammatory potential, as expressed as the E-DII, was associated with an increased risk of developing knee OA in this prospective US cohort. Such an association was mediated by BMI independent of total energy intake, suggesting an integrated role of inflammatory potential in dietary strategy for people at high risk of knee OA.

\begin{abstract}
Abbreviations
OA: Osteoarthritis; BMI: Body mass index; DIl: Dietary inflammatory index; SxOA: Symptomatic knee OA; OAl: Osteoarthritis Initiative; FFQ: Food Frequency Questionnaire; CRP: C-reactive protein; IL: Interleukin; TNF: Tumor necrosis factor; E-DIl: Energy-adjusted DIl; KL: Kellgren and Lawrence; ROA: Radiographic knee OA; PASE: Physical Activity Scale for the Elderly; GEE: Generalized estimating equations; OR: Odds ratio; Cl: Confidence interval
\end{abstract}

Acknowledgements

We would like to thank the OAI participants, OAI investigators, and OAI Clinical Center's staff for generating this publicly available dataset.

\section{Authors' contributions}

$\mathrm{QL}$ analyzed the data. QL, JH, NS, JL, and $\mathrm{YZ}$ were the major contributors in writing the manuscript. All authors interpreted the data and critically reviewed and revised the article for important intellectual content. All authors read and approved the final manuscript.

\section{Funding}

This work was supported by the Osteoarthritis Research Society International [Collaborative Scholarship 2017 to QL], the Beijing Joint Care Foundation [Young Investigator Scholarship 2017 to QL], and the National Natural Science Foundation of China [81902247 to QL]. The Osteoarthritis Initiative is a public-private partnership comprising five National Institutes of Health (NIH) contracts [N01-AR-2-2258, N01-AR-2-2259, N01-AR-2-2260, N01-AR-22261, and N01-AR-2-2262] funded by the NIH and conducted by the OAI Study Investigators. Private sector funding for the OAl is managed by the Foundation for the $\mathrm{NIH}$. The funding sources had no role in the design of the study; collection, analysis, and interpretation of the data; and in writing the manuscript.

Availability of data and materials

The datasets generated and/or analyzed during the current study are available in the OAl repository, https://nda.nih.gov/oai. 


\section{Ethics approval and consent to participate}

The institutional review board at each of the sites approved the study, and informed consent was obtained from all participants.

\section{Consent for publication}

\author{
Not applicable.
}

\section{Competing interests}

Dr. James R. Hébert owns a controlling interest in Connecting Health Innovations LLC, a company that has licensed the right to his invention of the dietary inflammatory index from the University of South Carolina in order to develop computer and smartphone applications for patient counseling and dietary intervention in clinical settings. Dr. Nitin Shivappa is an employee of Connecting Health Innovations LLC. The subject matter of this paper will not have any direct bearing on that work nor has that activity exerted any influence on this project. The authors declare that they have no competing interests.

\section{Author details}

${ }^{1}$ Arthritis Clinic and Research Center, Peking University People's Hospital, No.11 Xizhimen South Road, Xicheng District, Beijing 100044, China. ${ }^{2}$ Division of Rheumatology, Allergy and Immunology, Massachusetts General Hospital, Harvard Medical School, 55 Fruit St., Boston, MA 02114, USA. ${ }^{3}$ Cancer Prevention and Control Program, University of South Carolina, Columbia, SC, USA. ${ }^{4}$ Department of Epidemiology and Biostatistics, Arnold School of Public Health, University of South Carolina, Columbia, SC, USA. ${ }^{5}$ Capital University of Physical Education and Sports, Beijing, China. ${ }^{6}$ Department of Orthopedics, Xiangya Hospital, Central South University, Changsha, China.

Received: 22 May 2020 Accepted: 24 August 2020

Published online: 10 September 2020

\section{References}

1. Romagnolo DF, Selmin OI. Mediterranean diet and prevention of chronic diseases. Nutr Today. 2017;52:208-22.

2. Schulze MB, Martinez-Gonzalez MA, Fung TT, Lichtenstein AH, Forouhi NG. Food based dietary patterns and chronic disease prevention. BMJ. 2018;361: k2396.

3. Giugliano D, Ceriello A, Esposito K. The effects of diet on inflammation: emphasis on the metabolic syndrome. J Am Coll Cardiol. 2006;48:677-85.

4. Azadbakht L, Surkan PJ, Esmaillzadeh A, Willett WC. The Dietary Approaches to Stop Hypertension eating plan affects $C$-reactive protein, coagulation abnormalities, and hepatic function tests among type 2 diabetic patients. J Nutr. 2011;141:1083-8.

5. Cui $X$, Jin Y, Singh UP, Chumanevich AA, Harmon B, Cavicchia P, et al. Suppression of DNA damage in human peripheral blood lymphocytes by a juice concentrate: a randomized, double-blind, placebo-controlled trial. Mol Nutr Food Res. 2012;56:666-70.

6. Ruiz-Canela M, Zazpe I, Shivappa N, Hebert JR, Sanchez-Tainta A, Corella D, et al. Dietary inflammatory index and anthropometric measures of obesity in a population sample at high cardiovascular risk from the PREDIMED (PREvencion con Dleta MEDiterranea) trial. Br J Nutr. 2015;113:984-95.

7. Ramallal R, Toledo E, Martinez JA, Shivappa N, Hebert JR, Martinez-Gonzalez $M A$, et al. Inflammatory potential of diet, weight gain, and incidence of overweight/obesity: the SUN cohort. Obesity (Silver Spring). 2017;25:9971005.

8. Ramallal R, Toledo E, Martinez-Gonzalez MA, Hernandez-Hernandez A, Garcia-Arellano A, Shivappa N, et al. Dietary inflammatory index and incidence of cardiovascular disease in the SUN cohort. PLoS One. 2015;10: e0135221.

9. Boden S, Wennberg M, Van Guelpen B, Johansson I, Lindahl B, Andersson J, et al. Dietary inflammatory index and risk of first myocardial infarction; a prospective population-based study. Nutr J. 2017;16:21.

10. Thijssen E, van Caam A, van der Kraan PM. Obesity and osteoarthritis, more than just wear and tear: pivotal roles for inflamed adipose tissue and dyslipidaemia in obesity-induced osteoarthritis. Rheumatology (Oxford). 2015;54:588-600.

11. Neogi T, Zhang Y. Epidemiology of osteoarthritis. Rheum Dis Clin N Am. 2013;39:1-19.

12. Disease GBD, Injury I, Prevalence C. Global, regional, and national incidence, prevalence, and years lived with disability for 328 diseases and injuries for
195 countries, 1990-2016: a systematic analysis for the Global Burden of Disease Study 2016. Lancet. 2017;390:1211-59.

13. Roos EM, Arden NK. Strategies for the prevention of knee osteoarthritis. Nat Rev Rheumatol. 2016;12:92-101.

14. Green JA, Hirst-Jones KL, Davidson RK, Jupp O, Bao Y, MacGregor AJ, et al. The potential for dietary factors to prevent or treat osteoarthritis. Proc Nutr Soc. 2014;73:278-88.

15. Thomas S, Browne H, Mobasheri A, Rayman MP. What is the evidence for a role for diet and nutrition in osteoarthritis? Rheumatology (Oxford). 2018; 57(Suppl 4):iv61-74.

16. Hu FB. Dietary pattern analysis: a new direction in nutritional epidemiology. Curr Opin Lipidol. 2002;13:3-9.

17. Zheng $\mathrm{H}$, Chen $\mathrm{C}$. Body mass index and risk of knee osteoarthritis: systematic review and meta-analysis of prospective studies. BMJ Open. 2015;5:e007568.

18. Tabung FK, Smith-Warner SA, Chavarro JE, Wu K, Fuchs CS, Hu FB, et al. Development and validation of an empirical dietary inflammatory index. J Nutr. 2016;146:1560-70.

19. Dai Z, Niu J, Zhang Y, Jacques P, Felson DT. Dietary intake of fibre and risk of knee osteoarthritis in two US prospective cohorts. Ann Rheum Dis. 2017; 76:1411-9.

20. Block G, Hartman AM, Naughton D. A reduced dietary questionnaire: development and validation. Epidemiology. 1990;1:58-64.

21. Block G, Hartman AM, Dresser CM, Carroll MD, Gannon J, Gardner L. A databased approach to diet questionnaire design and testing. Am J Epidemiol. 1986;124:453-69.

22. Cavicchia PP, Steck SE, Hurley TG, Hussey JR, Ma Y, Ockene IS, et al. A new dietary inflammatory index predicts interval changes in serum highsensitivity C-reactive protein. J Nutr. 2009;139:2365-72.

23. Shivappa N, Steck SE, Hurley TG, Hussey JR, Hebert JR. Designing and developing a literature-derived, population-based dietary inflammatory index. Public Health Nutr. 2014:17:1689-96.

24. Shivappa N, Steck SE, Hurley TG, Hussey JR, Ma Y, Ockene IS, et al. A population-based dietary inflammatory index predicts levels of $\mathrm{C}$-reactive protein in the Seasonal Variation of Blood Cholesterol Study (SEASONS). Public Health Nutr. 2014;17:1825-33.

25. Shivappa N, Hebert JR, Rietzschel ER, De Buyzere ML, Langlois M, Debruyne $E$, et al. Associations between dietary inflammatory index and inflammatory markers in the Asklepios Study. Br J Nutr. 2015;113:665-71.

26. Tabung FK, Steck SE, Zhang J, Ma Y, Liese AD, Agalliu I, et al. Construct validation of the dietary inflammatory index among postmenopausal women. Ann Epidemiol. 2015;25:398-405.

27. Wirth MD, Shivappa N, Davis L, Hurley TG, Ortaglia A, Drayton R, et al. Construct validation of the dietary inflammatory index among African Americans. J Nutr Health Aging. 2017;21:487-91.

28. Willett WC, Howe GR, Kushi LH. Adjustment for total energy intake in epidemiologic studies. Am J Clin Nutr. 1997;65(Suppl 4):1220S-8S.

29. Martin KA, Rejeski WJ, Miller ME, James MK, Ettinger WH Jr, Messier SP. Validation of the PASE in older adults with knee pain and physical disability. Med Sci Sports Exerc. 1999;31:627-33.

30. Erikson R, Goldthorpe JH, Jackson M, Yaish M, Cox DR. On class differentials in educational attainment. Proc Natl Acad Sci U S A. 2005;102:9730-3.

31. Jackson M, Erikson R, Goldthorpe $J H$, Yaish M. Primary and secondary effects in class differentials in educational attainment: the transition to A-level courses in England and Wales. Acta Sociologica. 2007:50:211-29.

32. Liu X, Machado GC, Eyles JP, Ravi V, Hunter DJ. Dietary supplements for treating osteoarthritis: a systematic review and meta-analysis. Br J Sports Med. 2018;52:167-75.

33. Sacks FM, Obarzanek E, Windhauser MM, Svetkey LP, Vollmer WM, McCullough M, et al. Rationale and design of the Dietary Approaches to Stop Hypertension trial (DASH). A multicenter controlled-feeding study of dietary patterns to lower blood pressure. Ann Epidemiol. 1995;5:108-18.

34. Felson DT, Zhang Y, Anthony JM, Naimark A, Anderson JJ. Weight loss reduces the risk for symptomatic knee osteoarthritis in women. The Framingham Study. Ann Intern Med. 1992;116:535-9.

35. Riecke BF, Christensen R, Christensen P, Leeds AR, Boesen M, Lohmander LS, et al. Comparing two low-energy diets for the treatment of knee osteoarthritis symptoms in obese patients: a pragmatic randomized clinical trial. Osteoarthr Cartil. 2010;18:746-54.

36. Messier SP, Mihalko SL, Legault C, Miller GD, Nicklas BJ, DeVita P, et al. Effects of intensive diet and exercise on knee joint loads, inflammation, and 
clinical outcomes among overweight and obese adults with knee osteoarthritis: the IDEA randomized clinical trial. JAMA. 2013;310:1263-73.

37. Christensen P, Henriksen M, Bartels EM, Leeds AR, Meinert Larsen T, Gudbergsen $\mathrm{H}$, et al. Long-term weight-loss maintenance in obese patients with knee osteoarthritis: a randomized trial. Am J Clin Nutr. 2017;106:75563.

38. Hebert JR, Frongillo EA, Adams SA, Turner-McGrievy GM, Hurley TG, Miller $D R$, et al. Perspective: randomized controlled trials are not a panacea for diet-related research. Adv Nutr. 2016;7:423-32.

39. Goldring MB, Otero M. Inflammation in osteoarthritis. Curr Opin Rheumatol. 2011;23:471-8

40. Sokolove J, Lepus CM. Role of inflammation in the pathogenesis of osteoarthritis: latest findings and interpretations. Ther Adv Musculoskelet Dis. 2013;5:77-94.

41. Barbaresko J, Koch M, Schulze MB, Nothlings U. Dietary pattern analysis and biomarkers of low-grade inflammation: a systematic literature review. Nutr Rev. 2013;71:511-27.

42. Casas R, Sacanella E, Estruch R. The immune protective effect of the Mediterranean diet against chronic low-grade inflammatory diseases. Endocr Metab Immune Disord Drug Targets. 2014;14:245-54.

43. Livshits G, Zhai G, Hart DJ, Kato BS, Wang H, Williams FM, et al. Interleukin-6 is a significant predictor of radiographic knee osteoarthritis: the Chingford Study. Arthritis Rheum. 2009;60:2037-45.

44. Stannus O, Jones G, Cicuttini F, Parameswaran V, Quinn S, Burgess J, et al. Circulating levels of IL-6 and TNF-alpha are associated with knee radiographic osteoarthritis and knee cartilage loss in older adults. Osteoarthr Cartil. 2010;18:1441-7.

45. Jin X, Beguerie JR, Zhang W, Blizzard L, Otahal P, Jones G, et al. Circulating C reactive protein in osteoarthritis: a systematic review and meta-analysis. Ann Rheum Dis. 2015;74:703-10.

46. Hill AB. Observation and experiment. N Engl J Med. 1953;248(24):995-1001.

47. Hill AB. The environment and disease: association or causation? Proc R Soc Med. 1965;58:295-300

48. Shivappa N, Wirth MD, Murphy EA, Hurley TG, Hebert JR. Association between the Dietary Inflammatory Index (DII) and urinary enterolignans and C-reactive protein from the National Health and Nutrition Examination Survey-2003-2008. Eur J Nutr. 2019:58:797-805.

49. Shivappa N, Bonaccio M, Hebert JR, Di Castelnuovo A, Costanzo S, Ruggiero $\mathrm{E}$, et al. Association of proinflammatory diet with low-grade inflammation: results from the Moli-sani study. Nutrition. 2018;54:182-8.

50. Wallace IJ, Worthington $\mathrm{S}$, Felson DT, Jurmain RD, Wren KT, Maijanen $H_{\text {, }}$ et al. Knee osteoarthritis has doubled in prevalence since the mid-20th century. Proc Natl Acad Sci U S A. 2017;114:9332-6.

\section{Publisher's Note}

Springer Nature remains neutral with regard to jurisdictional claims in published maps and institutional affiliations.

Ready to submit your research? Choose BMC and benefit from:

- fast, convenient online submission

- thorough peer review by experienced researchers in your field

- rapid publication on acceptance

- support for research data, including large and complex data types

- gold Open Access which fosters wider collaboration and increased citations

- maximum visibility for your research: over $100 \mathrm{M}$ website views per year

At $\mathrm{BMC}$, research is always in progress.

Learn more biomedcentral.com/submissions 